\title{
Pulsed laser fabrication of 3D diamond detectors
}

\author{
Stefano Lagomarsino ${ }^{1}$, Giuliano Parrini, Silvio Sciortino \\ National Institute of Nuclear Physics (INFN), Via B. Rossi 1-3, 50019 Sesto Fiorentino (FI), Italy \\ Via Sansone 1, 50019 Sesto Fiorentino, Italy, \\ E-mail: lagomarsinoefi.infn.it, parriniefi.infn.it, sciortinoefi.infn.it
}

\section{Marco Bellini, Chiara Corsi, Federico Gorelli}

European Laboratory for Non-Linear Spectroscopy,

Via Nello Carrara 1, 50019 Sesto Fiorentino (FI), Italy

E-mail: bellinieino.it, corsielens.unifi.it, gorellielens.unifi.it

\section{Mario Santoro}

Istituto di Fisica Applicata N. Carrara-CNR (IFAC-CNR),

Via Madonna del Piano 10, 50019 Sesto Fiorentino (FI), Italy

E-mail: santorodlens.unifi.it

\begin{abstract}
3D detectors, whose electrodes extend perpendicularly to the sensor surface, represent one of the solution proposed for the challenges of radiation-harsh environments in high energy physics [1-4]. We report on the fabrication and characterization of prototypes of 3D diamond detector, which add to the 3D architecture the advantages of diamond as a sensor for tracking purposes. Two different laser sources, a Nd:YAG 1064 Q-switched laser with 8 ns pulse-width and a Ti:sapphire laser source with $30 \mathrm{fs}$ pulse duration have been used to fabricate arrays of graphitic columns in the bulk of a polycrystalline and a single crystal diamond sample. The columns are staggered and connected to graphitic combs which have been fabricated as well by laser irradiation and used as electric contacts. On each sample, an identical pattern of graphitic combs without columns (2D structure) has also been fabricated as a reference. The charge collection efficiency of each 3D sensor has been measured at different voltages and compared with the corresponding $2 \mathrm{D}$ structures. The much lower saturation voltages of the $3 \mathrm{D}$ sensors compared to those of the planar ones confirm that charge collection takes place at the columnar electrodes. Moreover, an efficiency of $100 \%$ is assured by the sensors fabricated with the fslaser source, while a loss in efficiency up to $30 \%$ is observed with the sensors fabricated with the ns-laser. The expected behaviour of $3 \mathrm{D}$ diamond sensors after strong radiation damage is discussed.
\end{abstract}

11th International Conference on Large Scale Applications and Radiation Hardness of Semiconductor Detectors

July 3-5, 2013

Florence, Italy

1

Speaker 


\section{Introduction}

In a conventional solid state radiation detector the track of the particles and the path followed by each generated charge drifting inside the active volume have about the same length, of the order of the sensor thickness. If the material becomes highly defective, which is the case in harsh radiation environments typical of the high luminosity collider experiments, the charge collection efficiency is limited, at most, to the ratio between the mean capture length and the sensor thickness [5].

A possible solution for the problem of the signal degradation due to radiation damage was proposed by Segal et al.[6]: the arrangement of thin, cylindrical electrodes only a few tens of microns apart, perpendicular to the sensor surface, in such a way that a substantially increased amount of the generated charges could be collected before trapping. The so called " $3 \mathrm{D}$ " concept has been developed and experimented in silicon detectors [1,2], for which this solution allows also the employment of correspondingly lower depleting voltages, due to the decreased distance between the polarizing electrodes.

Recently, the 3D approach has been also experimented in diamond detectors [3,4,7], which offer the possibility to exploit the long studied radiation hardness properties of this material $[8,9]$. The $3 \mathrm{D}$ columnar electrodes are fabricated by a pulsed laser technique. The diamond sample is irradiated by the laser beam and suitably moved in the direction of its thickness in such a way that the beam waist moves across the diamond bulk, leaving a graphitic conductive path [10].

The characteristics of the graphitic material depend, in these structures, principally on the laser pulse duration and wavelength, ranging from the femtoseconds $[7,10]$ to the nanoseconds $[3,4]$, and from the near UV [3,4] to the near IR [7,10]. Ultra-short laser pulses tend to require a lower energy per pulse to fabricate well-shaped structures with minimum damage of the surrounding material [11]. On the other hand, the resistivity of the graphitic material fabricated with ns-pulses is about ten times lower than that fabricated with the fs-ones, which is a strong advantage in view of the fabrication of low-noise electrodes for 3D pixel detectors [11].

In this article we report on the fabrication of 3D diamond sensors both from monocrystalline and from polycrystalline diamond, exploiting two different laser sources, in the nanosecond and in the femtosecond domains. The sensors have been tested for $\mathrm{Sr}^{90}$ betaparticle detection.

A model based on the degradation properties of the single crystal and polycrystalline diamond as observed in conventional planar sensors has also been developed in order to foresee the behavior of 3D diamond sensors after strong radiation fluences.

\section{Experimentals}

\subsection{Sample fabrication}

We employed two different pulsed laser sources: (i) a Q-switched Nd:YAG laser, operating at the $1064 \mathrm{~nm}$ wavelength with a $8 \mathrm{~ns}$ pulse width in the $10-40 \mu \mathrm{J}$ pulse energy range 
and a repetition rate from 1 to $5 \mathrm{kHz}$; (ii) a Ti:sapphire, $800 \mathrm{~nm}$ source with a pulse width of about $30 \mathrm{fs}$, an energy-per-pulse of about $5 \mu \mathrm{J}$ and a repetition rate of $1 \mathrm{kHz}$. While both the laser sources were employed in the fabrication of the 3D electrodes, all the superficial contacts were made engraving the diamond surface with the nanosecond Nd:YAG beam. We found [12] that superficial contacts made in this way yield a resistivity comparable to natural graphite and optimal geometric characteristics. In order to use both the sources in the fabrication of hybrid devices, they were employed in the same optical set-up.

In the present work we processed two diamond samples from Element 6 , single crystal and polycrystalline, respectively, with dimensions $5 \times 5 \times 0.5 \mathrm{~mm}^{3}$. Several different sensors, all about $1.5 \times 1.5 \mathrm{~mm}^{2}$ sized, were fabricated in each sample, with different geometrical characteristics.

Interdigitated Combs (IDC) sensors

Two interdigitated combs with a teeth-pitch of $160 \mu \mathrm{m}$ (in each comb), were engraved on a same side of the diamond sample; then the sample was reversed and two systems of staggered $500 \mu \mathrm{m}$-long, $100 \mu \mathrm{m}$ apart columns were fabricated, beginning from the superficial graphitization and proceeding towards the other side of the sample (see Fig 1a). The two combs were intended to support ground and bias polarization respectively, in a way that the charge collected in each cell of $160 \times 100 \mu \mathrm{m}^{2}$ size is collected by a single electrode at the center of the cell. This arrangement is quite simple to implement, but it is very sensitive to surface currents.

Opposite Side Combs (OSC) sensors

The two combs are engraved on the opposite sides of the sample, in a way that the projection of the first one onto the opposite side results interdigitated with the other one (see Fig. 2b). Then, two systems of columns are fabricated in a similar manner to the IDC sensors, but carefully avoiding the emergence of each column on the opposite side (in fact, stopping each column at about $50 \mu \mathrm{m}$ from the opposite surface). Finally, a second system of superficial teeth is added to each comb, covering the projection of the other comb on the same side, in order to make more uniform the field produced by the two graphitization patterns in the diamond bulk. Although this configuration is considerably much complex than the IDC, it avoids the application of the bias voltage to the same surface of a diamond sample, which proved to be cause of erratic discharges in previous preliminary experiments.
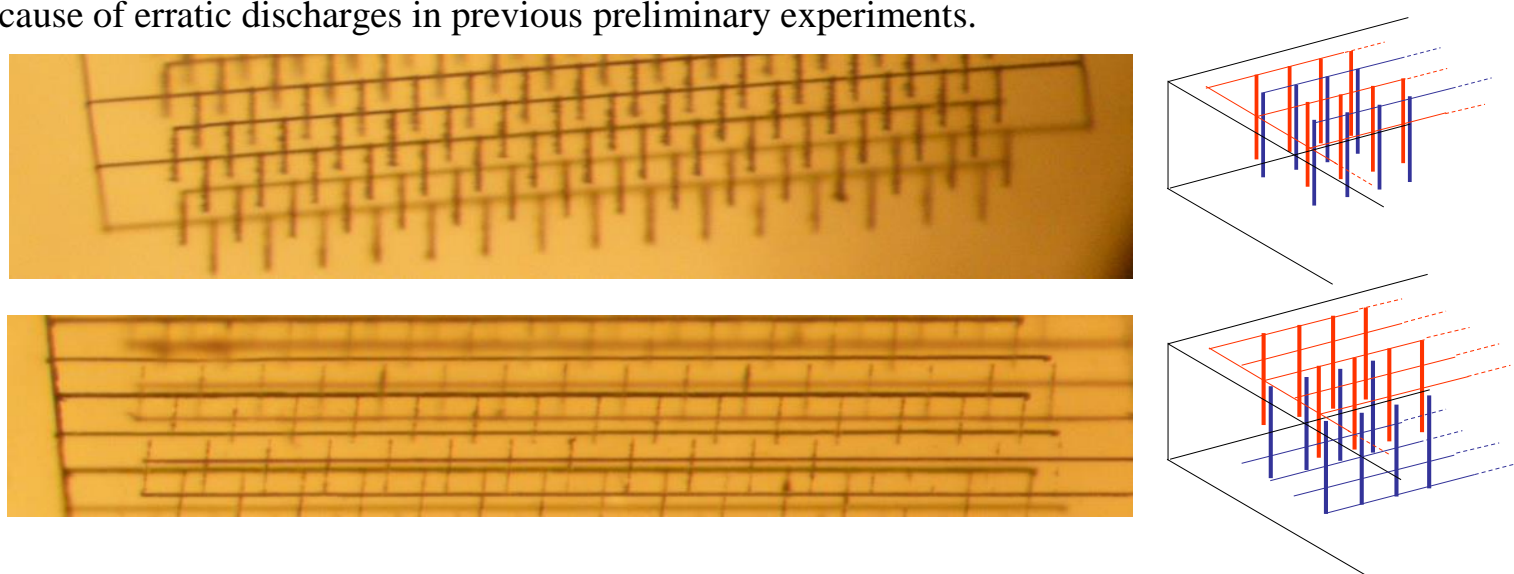

Figure 1. a) Interdigitated Comb (IDC) sensor, made with the fs-laser source. b) Opposite Side Comb (OSC) sensor, fabricated with the ns-laser. 


\section{Reference}

Two combs with teeth-pitch of $80 \mu \mathrm{m}$ were fabricated on the opposite sides of the samples, without columns, in order to compare the behavior of the 3D-sensors with that of conventional planar-ones, and to exclude the possibility that charge collection in 3D OSC devices takes place between the superficial graphitizations.

Six sensors were fabricated, four on the monocrystalline diamond (an IDC sensor made with the fs-laser, an IDC and an OSC made with the ns-one, and a reference sensor), and two on the polycrystalline (an OSC sensor fabricated with the nanosecond laser and the reference sensor).

\subsection{Sensors test}

The set-up employed in the measurement of the collection efficiency of the sensors has been described in detail elsewhere [13]. The signal is pre-amplified, formed with a peaking time of $1.4 \mu \mathrm{s}$ and then acquired by means of a 12 bit ADC. The system was calibrated in order to obtain an estimate of the number of charge carriers collected from the maximum amplitude of the formed signal, obtaining a constant ratio of $220 \mathrm{e} / \mathrm{mV} \pm 15 \%$. The electrons of the ${ }^{90} \mathrm{Sr}$ spectrum having enough energy to pass through the sample and the coating of the trigger plastic scintillator are expected to generate about 36 electron-hole couples per micron in the diamond, in such a way that full collection should consist in an average signal of 18000 electrons (within the calibration error).

Spectra have been acquired for each sensor and for each value of the bias voltage. In the case of the polycrystalline diamond sensors the samples were primed by irradiation with a 10 $\mathrm{mCi} \mathrm{Sr}^{90}$ source for several minutes, as usual for polycrystalline sensors [14], in order to maximize the response, and then kept in the dark after priming and during measurements.

\section{Results}

In figure 2a, the average signal from each sensor fabricated in the monocrystalline sample is plotted vs. the bias voltage (positive voltage, as for the negative voltage the plot is practically symmetric). The main features emerging are the following:

- Full collection is attained both with the reference and with the 3D IDC fs-sensor, but $90 \%$ of the saturation signal for the $3 \mathrm{D}$ sensor is reached at about $3 \mathrm{~V}$, to be compared with the $30 \mathrm{~V}$ of the reference sensor. This is due to the higher field values obtained, for the same bias voltage, in the 3D structures, and confirms that charge collection takes place along the vertical graphitic electrodes, not at the superficial graphitic combs.

- The ns-sensors, irrespective of their geometry (IDC or OSC), have a charge loss of about $30 \%$ although the saturation voltage is about the same as for the 3D fs-sensor.

Figure $2 \mathrm{~b}$ shows the pulse height spectra of the monocrystalline sensors at saturation voltage. For the 3D ns-sensors it is apparent that the distributions does not follow the expected Landau profile, but are given by the superposition of (at least) two components. The data are fitted with the superposition of two Landau profiles, convoluted with a normal noise distribution 
given by the pedestal at zero collected charge (false events). This results in a ratio $r$ between the lower and the higher component which is $35 \%$ for the OSC and $17 \%$ for the IDC ns-sensor.

Figures $3 \mathrm{a}$ and $3 \mathrm{~b}$ give the same kind of information of fig. 2, but corresponding to the polycrystalline sensors. The saturation signal of the reference sensor is only about $30 \%$ of the full collection and the saturation voltage seems not completely attained even at a bias voltage of $600 \mathrm{~V}$ (see the inset of figure 3a). This is expected for a polycrystalline sensor, where the highly defective material strongly limits the capture time of the traps and the recombination centers.
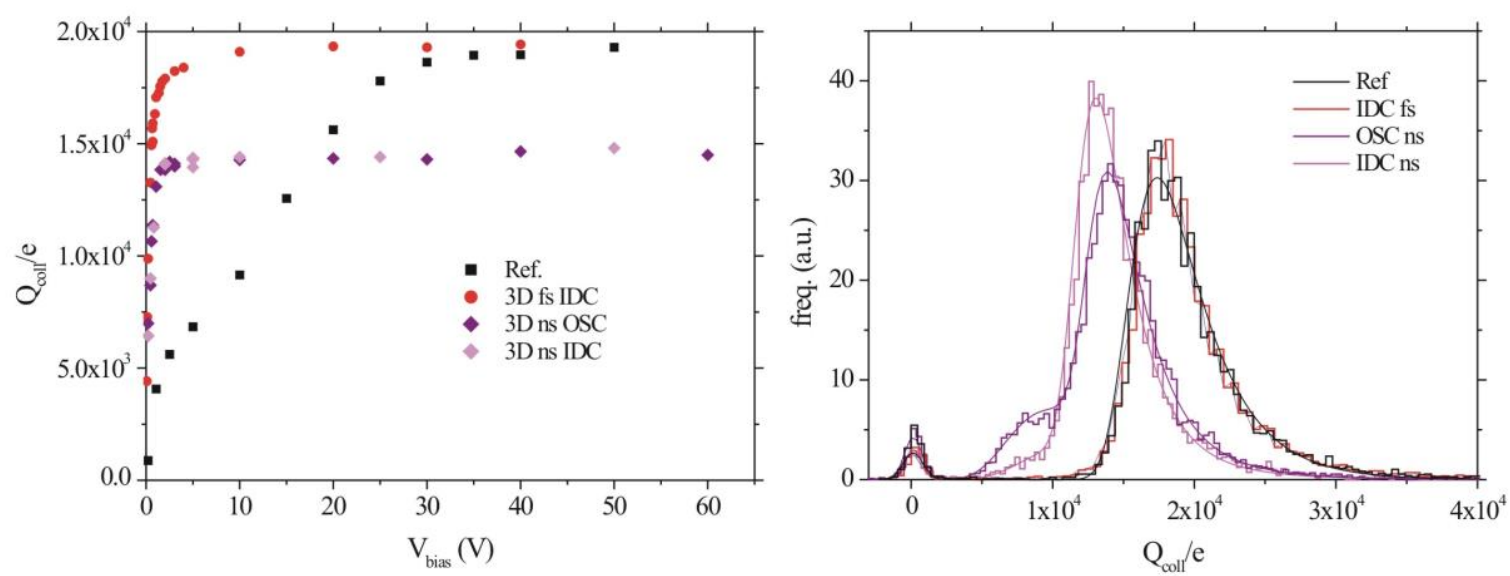

Figure 2. a) Average collected charge versus bias voltage for the three sensors fabricated on the single crystal diamond sample; b) pulse height spectrum of the sensors biased at the saturation voltage
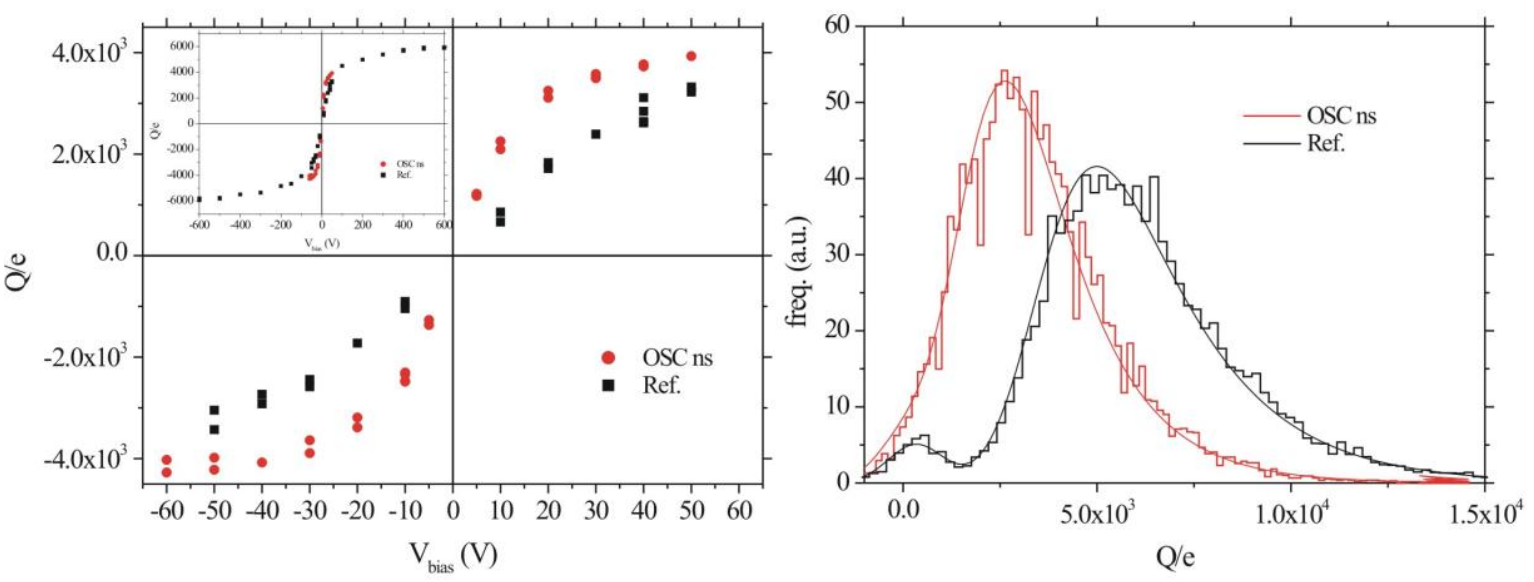

Figure 3. a) Average collected charge versus bias voltage for the 3D- (red points) and for the 2D-detector (black points) fabricated on the polycrystalline diamond sample; b) pulse height spectrum of the sensors biased at the saturation voltage. The 3D sensor could not be tested at voltages higher than about $70 \mathrm{~V}$, because erratic discharges determine a noise level much higher than the signals. The 2D sensor was tested up to $600 \mathrm{~V}$ of bias voltage (see the inset of fig 3a).

In fact, for the best of these samples [15], also at the saturation velocity, attainable only with a polarization field $\mathrm{E}>>1 \mathrm{~V} / \mu \mathrm{m}$, no more than about $50 \%$ of the generated charge could be 
collected before trapping. The 3D ns-sensor, on the other hand, reach the saturation signal only at about $50 \mathrm{~V}$, confirming that the columnar electrodes are effective in charge collection, but the maximum collected charge is about $30 \%$ lower than that obtained with the reference sensor, coherently with the behavior of the 3D ns-sensors fabricated in the monocrystalline diamond.

\section{Discussion}

From the investigation of the experimental results, a remarkable conclusion emerges: 3D sensors fabricated with the fs-laser source attain $100 \%$ of collected charge $(18,000$ electrons in our $500 \mu \mathrm{m}$-thick diamond) with only a few volts of bias.

Comparing this performance with the poorer efficiency of the ns-sensors, ns-laser sources seems to be disqualified for fabrication of $3 \mathrm{D}$ diamond sensors. As a matter of fact, the characteristics of the graphitic materials created with the two kinds of laser source suggest more careful approach to the final implementation of these of sensors.

Up to now [11], there is a difference of one order of magnitude between the resistivity of the graphitic material made with the ns-laser source $(\sim 60 \mathrm{~m} \Omega \mathrm{cm})$ and with the fs-one $(\sim 800$ $\mathrm{m} \Omega \mathrm{cm}$ ), which determines, with the current values of the column cross sectional areas, a resistance of about $4 \mathrm{k} \Omega$ and $60 \mathrm{k} \Omega$ for the two kind of laser source, respectively. At the moment, all the research groups which are working with this kind of sensors are testing clusters of tens to hundreds of columns in parallel (see ref.[16] and the present work), with shaping times of the order of 1-2 $\mu$ s. In these conditions, the Johnson noise introduced by the input resistance is negligible, but in a pixel detector, working with signals from single columns, and with shaping times of the order of a few tens of nanoseconds like those employed in the current high energy physics experiments, a factor 15 in the series resistance could determine a factor 4 in the net signal-to-noise ratio, with a possible degradation of the performance well above the $30 \%$ signal loss highlighted in figure 2 a for the ns-sensors.

The reasons behind this signal loss deserve careful attention.

From inspection of figure $2 \mathrm{~b}$, both the IDC and (more prominently) the OSC signal distribution result from the superposition of two distinct signals. If fitted with two different Landau profiles, the less prominent represents 20\% (for the IDC) and 35\% (for the OSC) of the overall number of events, respectively, while the signal peak position of the weaker distribution is about $13 \%$ lower than that of the stronger one for the IDC and $28 \%$ for the OSC sensor. The simpler way to interpret these distributions is to attribute them to the missing or "aborted" columns evidenced by microscopic inspection (see fig.1, lower side). Each missing column, in fact, identifies a region in the diamond bulk which is polarized less efficiently, either by the surrounding column or by the superficial contacts, determining a loss of signal. On the other hand, even considering only the component with the higher peak, it is apparent that the average signal does not reach the full collected charge exhibited by the 3D sensors made with the fslaser. The Raman analysis of the material modified by ns- and fs-laser radiation[11] suggests a possible reason for that signal loss. In fact, ns-laser-made columns appear to be surrounded by a thin $(\sim 5 \mu \mathrm{m})$ layer of nanostructured $\mathrm{sp}^{3}$-carbon, highly resistive but also highly defective, supposedly trapping the charge carriers before they can reach the conductive electrode. This determines a percentage loss given by the ratio between the voltage drop across the defective 
layer and the bias voltage. If this is the reason of the signal degradation, a suitable annealing could solve this problem determining either the graphitization of this defective layer (making it conductive) or the recovering of the diamond crystalline structure[17].

\section{Behavior of 3D detectors after heavy irradiation, projections}

As an essential development of the present work, we are planning to expose our sensors to increasingly high level of radiation fluences, in order to observe the resulting signal degradation and to test the suitability of 3D diamond detectors to the challenges of radiation-harsh environments. In the meantime, we have simulated the expected behavior of a mono- or polycrystalline 3D sensor with electrode geometries equivalent to those employed in the present work, basing our calculation on the observed degradation of the signal obtained in experiments with conventional planar diamond sensors[8,9].

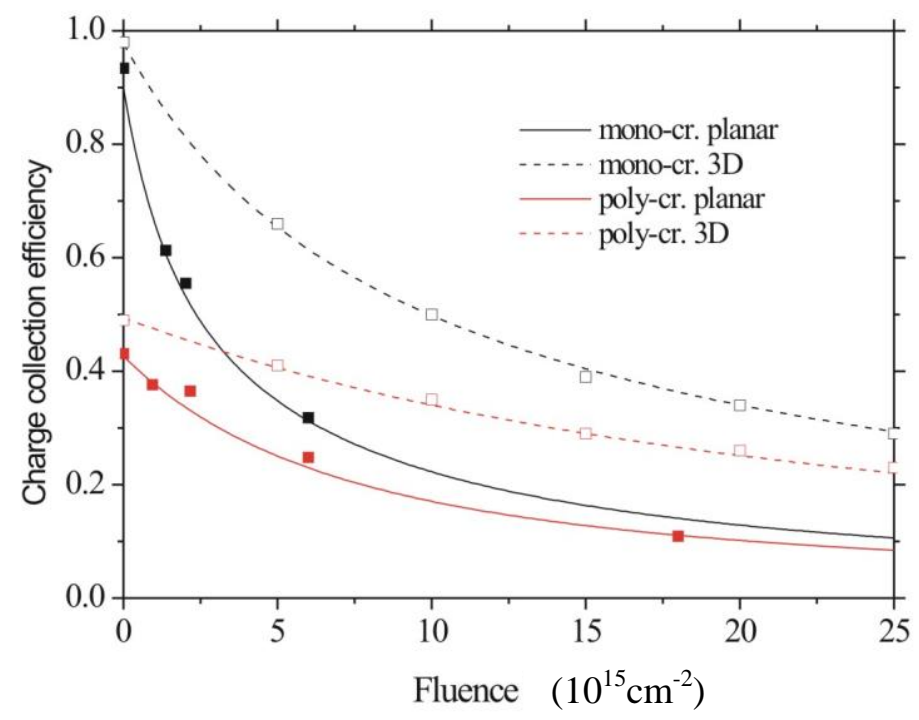

Figure 4. Full markers: charge collection efficiency of mono- (black) and poly-crystalline (red) planar diamond detectors, as a function of the fluence of $24 \mathrm{GeV}$ protons. Empty markers (and dotted line), projection, based on the behavior of the planar configuration, of the efficiency of 3D detectors.

For the single crystal diamond, we have supposed that the inverse capture lifetime of each kind of charge carrier is given adding to the undamaged diamond value $1 / \tau_{0}$ a term $a \times \phi$ proportional to the radiation fluence $\phi$, and adjusting the proportionality constant $a$ to fit the correspondence between fluence and charge collection efficiency. For the polycrystalline one, we have followed a similar hypothesis, but assuming that only one kind of carriers (presumably holes), in unirradiated polycrystalline diamond, has a lifetime long enough to contribute to the overall signal (for the justification of this hypothesis, see ref. [15] and related references). At this point, having an available expression for the capture time, we calculated the electric field produced by an array of cylindrical infinite electrodes with the geometry of our sensors, from which, given mobility and saturation velocity of the charge carriers, we performed a MonteCarlo simulation of the trajectories of the generated charges in the bulk diamond and of the 
charge induced on the electrodes by the motion of the carriers, hampered by the capture processes.

The results of our calculations are shown in figure 4, where the charge collection efficiency of single crystal and polycrystalline conventional $500 \mu \mathrm{m}$-thick diamond sensors are plotted as functions of proton MIP fluence, together with the projections of the behavior of 3D sensors of equal thickness. In the fluence range $1-2 \cdot 10^{16} \mathrm{~cm}^{-2}$, foreseen at the vertex of SLHC after 5 years, monocrystalline sensors should collect more than 7000 electrons, with a still negligible dark current (as typical of damaged diamond sensors) at room temperature. Noticeably, at the same fluence, the performance of the polycrystalline sensors could be only $25 \%$ lower.

\section{References}

[1] C. Da Vià, et al., Nuclear Instruments \& Methods in Physics Research A (2013), http://dx.doi.org/10.1016/j.nima.2013.05.048

[2] S.Grinstein,et al.,Nuclear Instruments \& Methods In Physics ResearchA (2013), http://dx.doi.org/10.1016/j.nima.2013.03.064

[3] Alexander Oh, Benoit Caylar, Michal Pomorski, Thorsten Wengler, A novel detector with graphitic electrodes in CVD diamond, Diamond \& Related Materials 38 (2013) 9-13

[4] Benoît Caylar, Michal Pomorski, and Philippe Bergonzo, Laser-processed three dimensional graphitic electrodes for diamond radiation detectors, Appl. Phys. Lett. 103, 043504 (2013);

[5] Karl Hecht, Zum Mechanismus des lichtelektrischen Primärstromes in isolierenden Kristallen, Zeitschrift für Physik, 1932, Volume 77, 3-4, pp235-245,

[6] J Segal S. I. Parker C. J. Kenney. A proposed new architecture for solid-state radiation detectors, Nucl. Instr. and Meth. A 395 pp 328-343 (1997)

[7] S. Lagomarsino et al., 3D Diamond detectors: charge collection efficiency of graphitic electrodes, in press in Applied Physics Letters.

[8] H. Kagan and W. Trischuk, Radiation Sensors for High Energy Physics Experiments, in CVD Diamond for Electronic Devices and Sensors, R. Sussman ed., 2009 John Wiley \& Sons Ltd

[9] D. Asnar, et al., The RD42 Collaboration, Diamond pixel modules, Nucl. Instr. \& Meth A 636 (2011) S125-S129

[10] T.V. Kononenko, M.S. Komlenok, V.P. Pashinin, S.M. Pimenov, V.I. Konov, M. Neff, V. Romano, W. Lüthy, Femtosecond laser microstructuring in the bulk of diamond, Diamond \& Related Materials 18 (2009) 196-199, doi:10.1016/j.diamond.2008.07.014

[11] S. Lagomarsino et al., Raman-imaging characterization of laser-made electrodes for 3D diamond detectors, submitted to Diamond and Related Materials

[12] G. Parrini, F. Fabbrizzi, S. Lagomarsino, L. Nunziati, S. Sciortino, A. Scorzoni, Laser graphitization for polarization of diamond sensors, PoS(RD11)017, 10th International Conference on Large Scale Applications and Radiation Hardness of Semiconductor Detectors Firenze, Italy July 6-8, 2011

[13] S. Sciortino, S. Lagomarsino, F. Nava, Silicon Carbide for High Signal to Noise Ratio MIPs Detection From Room Temperature to 80 oC, IEEE Transactions on Nuclear Science, vol. 56, issue 4, pp. 2538-2542, 2009,doi:10.1109/TNS.2009.2023848

[14] S. Mersi, E. Borchi, M. Bruzzi, R. D'Alessandro, S. Lagomarsino, S. Sciortino, A study of charge collection processes on polycrystalline diamond detectors, Nuclear Instruments and Methods in Physics Research A 530 (2004) 146-151, doi:10.1016/j.nima.2004.05.063

[15]E.Borchi, S.Lagomarsino, S.Mersi, S.Sciortino. Model of carrier dynamics in CVD Diamond detectors. Physical Review B. 71104103 (2005)

[16] Iain Haughton, 3D Diamond Prototype Studies, Diamond and 3D - Detectors for the Luminosity Frontier, IOP half day meeting, University of Manchester, 26 June 2013, https://indico.hep.manchester.ac.uk/conferenceTimeTable.py?confId=4031\#20130626

[17] R. Kalish, A. Reznik, K.W. Nugent, S. Prawer, The nature of damage in ion-implanted and annealed diamond, Nuclear Instruments and Methods in Physics Research B 148 (1999) 626-633. 